\title{
Safety Verification of SEITR Epidemic Model on Recombination HIV and Hepatitis B Virus using Taylor Model
}

\author{
Asmudik, Dieky Adzkiya, Mardlijah and Hariyanto
}

\begin{abstract}
Human Immunodeficiency Virus (HIV) is an AIDS (Acquired Immuno Deficiency Syndrome) virus that attacks the immune system for which there is no cure. When the immune system has decreased, it is prone to diseases such as Hepatitis $B$ disease. To reduce the error value of the number of subpopulations, we use an interval approximation. One of the simulation calculations that the number of variables initially intervals is Taylor model. Taylor's model can be used to verify that the number of people infected with HIV and Hepatitis B will not exceed the specified number of unsafe sets. To calculate the set of states that are reached by the system over a certain period of time, given the initial conditions and parameters. The initial condition is divided into three scenarios, an affordable set of states, safety verification can be done. As a result of the safety verification of the three scenarios provided there is no set of states that are not safe, so the results of all three scenarios are safe.
\end{abstract}

Index Terms-Recombination of HIV and Hepatitis B viruses, Taylor models, safety verification.

\section{INTRODUCTION}

$\mathbf{M}$ ANY methods can solve a system model such as Runge Kutta. But most of these methods require a value from the initial condition of a single value. This contradicts the fact that most subpopulations whose single value is uncertain. One method that the initial condition could be intervals is Taylor model. The initial condition of the model calculated using Taylor model can be both interval and single. This reduces the error value and results in a value that is close to the actual value. System verification can be done in a model. System verification aims to determine the state of the variables, parameters and changes that occur in the specified system conditions. Verification completion can use a software, and one of the software used to verify the model or system is flow star. Flow star is one of the applications that simulates the model of the system using Taylor model. Often pandemic diseases include HIV and Hepatitis B. Researchers here want to verify the complexity of HIV and Hepatitis B recombination, whether within 5 years, the disease becomes an outbreak or not. Based on the above description the purpose of this study is to analyze the safety verification results of SEITR epidemic model on the recombination of HIV and Hepatitis B virus with Taylor model.

The authors are with the Department of Mathematics, Institut Teknologi Sepuluh Nopember, Kampus ITS Sukolilo-Surabaya 60111, Indonesia e-mail: asmudik999@gmail.com, dieky@matematika.its.ac.id, mardlijah@matematika.its.ac.id, hariyanto@matematika.its.ac.id.

Manuscript received September 2, 2020; accepted January 20, 2021.
Previous research related to safety verification is [1]. In the study, the authors presented a case study of a simple hybrid multi basalt control system that switches to different preset insulin delivery rates across different ranges of glucose levels. The study used the Dalla-Man model to model the patient's physiology and the hybrid automaton model of the controller. The purpose of that research is to verify that blood glucose levels remain in a safe range overnight using flow* software. They used 2 different control strategies defined by a set of insulin levels. And the result of the study is that strategy I and strategy II do not cause hypolycemia and ketoacidosis, but their blood glucose is not in the euglycemic range. While blood glucose should be in the euglycemic range.

\section{Models AND PRELIMINARIES}

\section{A. Mathematical Model}

The model in this paper is a mathematical epidemic model constructed of type SEITR (Susceptible Exposed Infected Treatment Recovery). The model presented the progression of recombinant spread of HIV and Hepatitis B viruses. The population in the model was divided into 10 subpopulations. The subpopulations are the subpopulations of Susceptible $\left(S_{1}\right)$, Exposed HIV $\left(E_{1}\right)$, Exposed hepatitis B $\left(E_{2}\right)$, Infected HIV $\left(I_{1}\right)$, Infected Hepatitis B $\left(I_{2}\right)$, Infected AIDS $\left(I_{3}\right)$, Infected recombination HIV and hepatitis B $\left(I_{4}\right)$, Treatment HIV $\left(T_{1}\right)$, Treatment hepatitis B $\left(T_{2}\right)$, Recovery $(R)$ [2].

$$
\begin{aligned}
\frac{d S}{d t} & =A-\left(\beta_{1} I_{1}+\beta_{2} I_{2}+\mu\right) S \\
\frac{d E_{1}}{d t} & =\beta_{1} S I_{1}-\left(\gamma_{1}+\mu\right) E_{1} \\
\frac{d E_{2}}{d t} & =\beta_{2} S I_{2}-\left(\gamma_{2}+\mu\right) E_{2} \\
\frac{d I_{1}}{d t}= & \gamma_{1} E_{1}-\left(\theta+a_{1}+\mu\right) I_{1}+\left(1-c_{1}\right) \psi_{1} T_{1} \\
\frac{d I_{2}}{d t}= & \gamma_{2} E_{2}-\left(\omega_{1} I_{3}+a_{2}+\mu\right) I_{2}+\left(1-c_{2}\right) \psi_{2} T_{2} \\
\frac{d I_{3}}{d t}= & \theta I_{1}-\left(\omega_{1} I_{2}+a_{2}+\mu\right) I_{3}+\left(1-c_{3}\right) \psi_{3} T_{1} \\
\frac{d I_{4}}{d t}= & \omega_{1} I_{3} I_{2}+\omega_{2} I_{2} I_{3}-\left(a_{4}+\mu\right) I_{4}+\left(1-c_{4}\right) \psi_{4} T_{1} \\
\frac{d T_{1}}{d t}= & a_{1} I_{1}+a_{3} I_{3}+a_{4} F_{4}-\left(1-c_{1}\right) \psi_{1} T_{1}-\left(1-c_{3}\right) \\
& \quad-\left(1-c_{4}\right) \psi_{4} T_{1}-c_{4} \psi_{2} T_{2}-c_{1} \psi_{1} T_{1}-c_{3} \psi_{3} T_{1} \\
& \quad-\mu T_{1}
\end{aligned}
$$




$$
\begin{aligned}
& \frac{d T_{2}}{d t}=a_{2} I_{2}-\left(1-c_{2}\right) \psi_{2} T_{2}+c_{4} \psi_{4} T_{1}-\mu T_{2}-c_{2} \psi_{2} T_{2} \\
& \frac{d R}{d t}=c_{1} \psi_{1} T_{1}+c_{3} \psi_{3} T_{1}+c_{2} \psi_{2} T_{2}-\mu R
\end{aligned}
$$

where $S$ represents the number of susceptible individuals, $E_{1}$ represents the number of exposed HIV, $E_{2}$ represents the number of hepatitis B, $I_{1}$ represents the number of individuals infected by HIV, $I_{2}$ represents the number of individuals infected by hepatitis B, $I_{3}$ represents the number of individuals infected by AIDS, $I_{4}$ represents the number of individuals infected by HIV and hepatitis B, $T_{1}$ represents the number of individuals being treated for HIV, $T_{2}$ represents the number of individuals being treated for hepatitis $\mathrm{B}, R$ represents the number of recovered individuals.

The parameters used are as follows: $A$ denotes the number of births, $\mu$ denotes the death rate, $\beta_{i}$ denotes the rate of contact between susceptible and infected individuals for $i=1,2, \gamma_{i}$ denotes the rate of exposed individuals that entered the infected subpopulation for $i=1,2, \theta$ denotes HIV and AIDS transmission rate, $a_{j}$ denotes the treatment rate for $j=1,2,3,4, \omega_{i}$ denotes the contact rate between HIV-infected infividuals and individuals infected by hepatitis B virus for $i=1,2, c_{j}$ denotes the opportunity for the individual to experience a better change in condition due to treatment for $j=1,2,3,4$ and $\psi_{j}$ denotes the individual healing after treatment for $j=1,2,3,4$.

\section{B. Safety Verification}

The safety has the specification "no unsafe state can be reached". Safety verification issues can be written, given the $n$-dimensional dynamic system, the initial set of $X_{0} \in R^{n}$, unsafe set $U \subseteq R^{n}$, and time horizon $T>0$. The safety verification issue is proving that $\operatorname{Reach}_{u}\left(X_{0},[0, T]\right) \cap U=\emptyset$. In safety verification, we can overapproximate the reachable states. If no unsafe state can be reached, then the system is safe. The nature of safety is very important for hybrid systems and verification can be done by calculating reachability in the hybrid space state.

\section{Taylor's Model}

Taylor's model was originally developed by Berz and Makino to provide over-approximation for continuous functionality. Taylor models (TM) can be applied to overapproximation for flowpipes.

Definition 1 ([3]): Taylor's model is denoted by a pair $(p, I)$ where $p$ is a polynominal on the $\vec{x}$ variable set, starting in the domain interval $D$, and $I$ is the remaining interval. TM may also be worth a real-value TM vector, or in a way both $p$ and $I$ are vector-valued and have the same dimensions.

\section{RESUlts AND Discussions}

In this paper, three scenarios were used. The scenario is used to find out if hiv virus recombination disease and hepatitis B within a certain period will be an outbreak or not. The initial condition of scenario 1 is adjusted to the data taken from the previous model [2] and the unsafe condition is obtained from twice the initial condition with the focus of

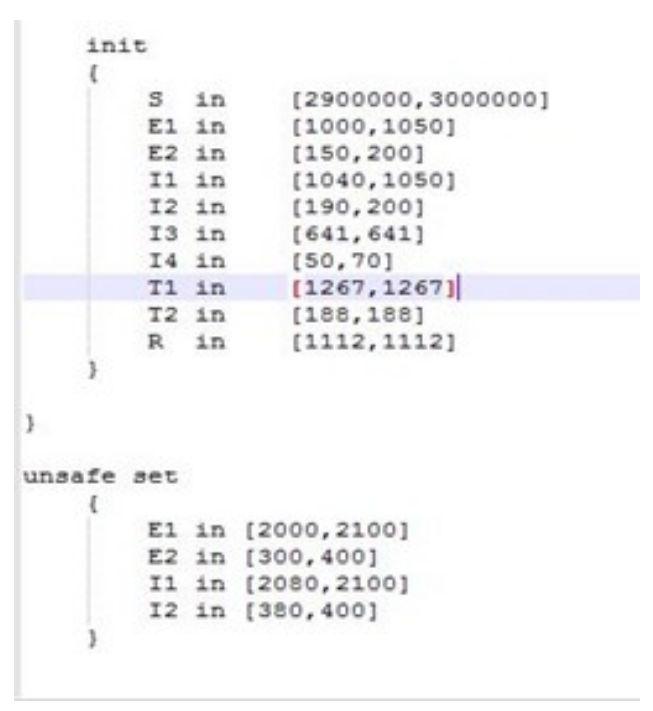

Fig. 1. The initial condition of the first scenario.

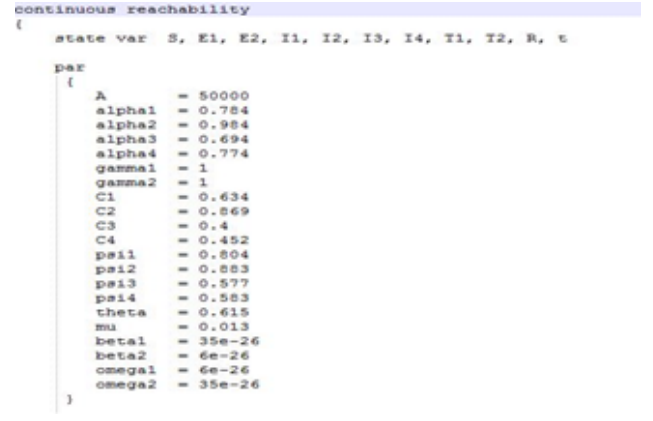

Fig. 2. System parameters.

research sub-population Exposed HIV ( $\left.E_{1}\right)$, Exposed hepatitis B $\left(E_{2}\right)$, Infected HIV $\left(I_{1}\right)$, Infected Hepatitis B $\left(I_{2}\right)$. The initial condition of the first scenario and its unsafe value can be seen in Fig. 1.

The initial condition of the second scenario is taken from the initial condition of the first scenario but the observed sub population is $20 \%$. The initial condition of the third scenario is taken from the initial condition of scenario 1 but the observed sub population is lowered by $20 \%$ and unsafe conditions are still obtained from the initial two conditions. Numerical completion to optimize the system in this mass is completed using flow star with parameter value can be from [2], along with the parameter value of the system from this research.

After a safety trait verification calculation using Flow* is obtained, the $E_{1}$ for the first scenario from start to finish down. The number $E_{1}$ is down from the initial number of 1000-1050 to close to the value of 0 . The image shows the number does not reach twice the initial amount. Therefore, the result is that the system satisfies the safety specification or the system is safe. The $E_{1}$ plot in the first scenario can be seen in Fig. 3.

The results of the next research are sub population $E_{2}$. The resulting plot for $E_{2}$ can be seen in Fig. 4 .

The result of the image can $E_{2}$ from start to finish down. The $E_{2}$ is down from the initial number of $150-200$ to close to the value of 0 . The image shows the number does not 


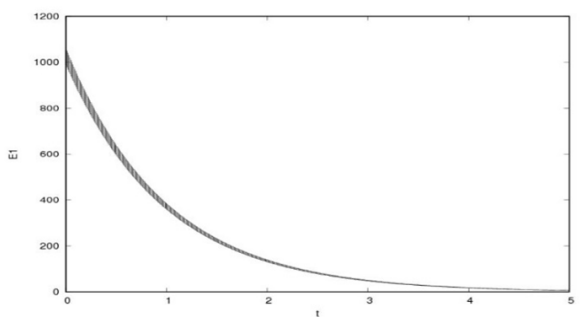

Fig. 3. The result of $E_{1}$ plot in the first scenario with a time of five years.

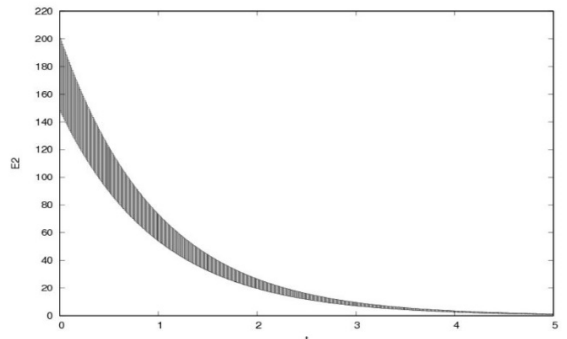

Fig. 4. The result of $E_{2}$ plot in the first scenario with a time of five years.

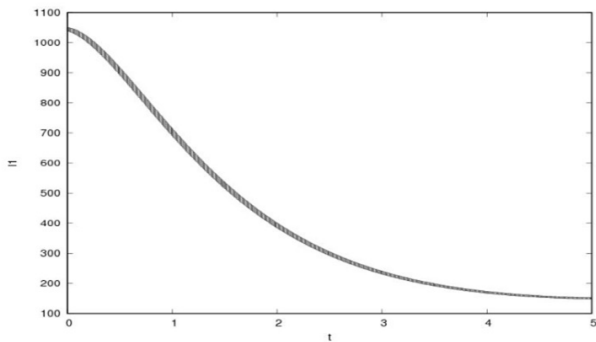

Fig. 5. The result of $I_{1}$ plot in the first scenario with a time of five years.

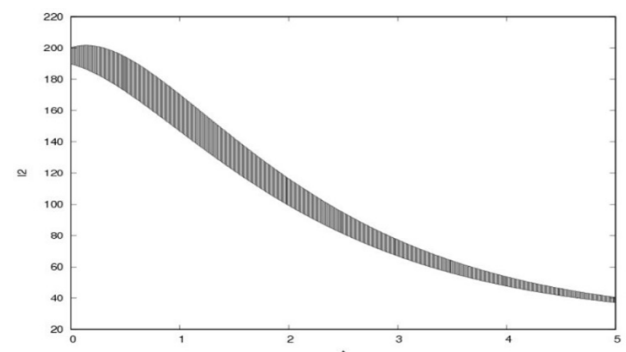

Fig. 6. The result of plot $I_{2}$ in the first scenario with a time of five years.

reach twice the initial amount, therefore the system is safe. The resulting plot of $I_{1}$ can be seen in Fig. 5 .

The result of the image can $I_{1}$ from start to finish down. The number $I_{1}$ the population is down from the initial number of $1140-1150$ to $150-155$. The image shows the number does not reach twice the initial amount, therefore the system is safe. The resulting plot $I_{2}$ can be seen in Fig. 6 .

The results of the image can be seen that $I_{2}$ isinitially constant a few months ago until the end went down. The number $I_{2}$ initially $190-200$ a few months ago dropped to the end of the year to $40-45$. The image shows the number does not reach twice the initial amount, therefore the system

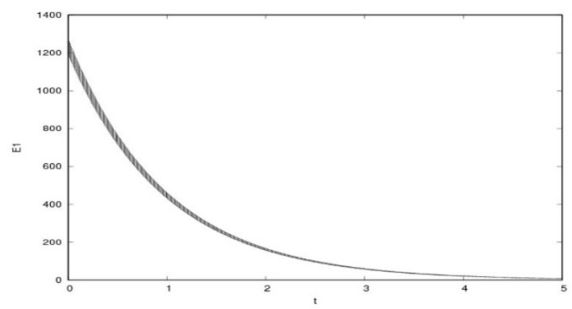

Fig. 7. The result of $E_{1}$ plot in the second scenario with a time of five years.

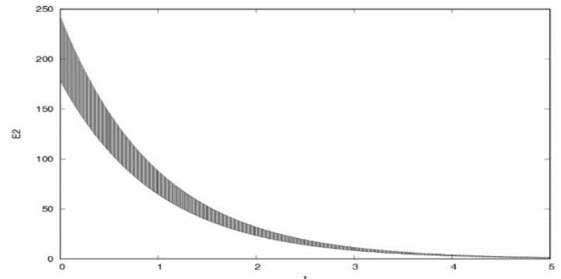

Fig. 8. The result of $E_{2}$ plot in the second scenario with a time of five years.

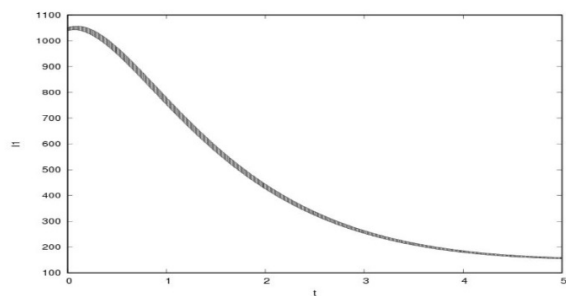

Fig. 9. The result of $I_{1}$ plot in the second scenario with a time of five years.

is safe. The second check is in the second scenario. The result of $E_{1}$ plot in the second scenario can be seen in Fig. 7.

The result of the image is that $E_{1}$ from start to finish down. The $E_{1}$ is down from the initial number of $1200-1260$ to close to the value of 0 . The image shows the number does not reach twice the initial amount, therefore the system is safe. The result of $E_{2}$ plot in the second scenario can be seen in Fig. 8.

The result of the image can $E_{2}$ from start to finish down. The number $E_{2}$ is down from the initial number of 180-240 to close to the value of 0 . The image shows the number does not reach twice the initial amount, therefore the system is safe. The result of $I_{1}$ plot in the second scenario can be seen in Fig. 9.

The result of the image can $I_{1}$ from start to finish down. The $I_{1}$ the number of people down from the beginning was $1140-1150$ to $160-165$. The image shows the number does not reach twice the initial amount, therefore the system is safe. The result $I_{2}$ the second scenario can be seen in Fig. 10 .

The result of the image can be $I_{2}$ initially only rose slightly a few months ago until the end of the fall. The $I_{2}$ initially 190200 rose slightly in the months to 195-210 then dropped by the end of the year to 40-45. The image shows the number does not reach twice the initial amount, therefore the system is safe. The third check is in scenario 3, the result of the plot $E_{1}$ the third scenario can be seen in Fig. 11.

The result of the image can $E_{1}$ from start to finish down. The $E_{1}$ is down from a starting number of $800-840$ to close 


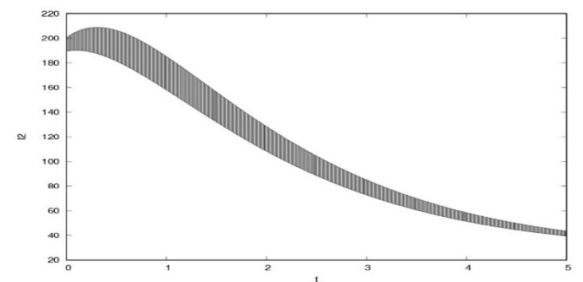

Fig. 10. The result of $I_{2}$ plot in the second scenario with a time of five years.

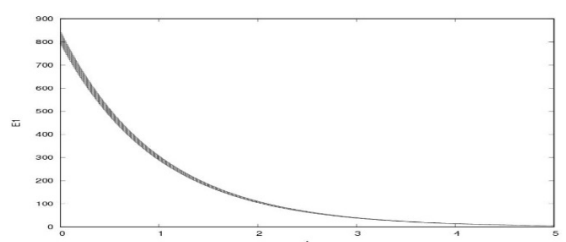

Fig. 11. The result of $E_{1}$ plot in the third scenario with a time of five years.

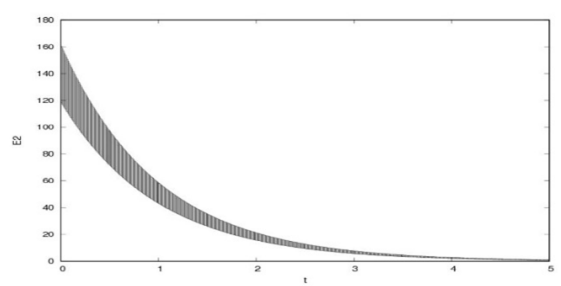

Fig. 12. The result of $E_{2}$ plot in the third scenario with a time of five years.

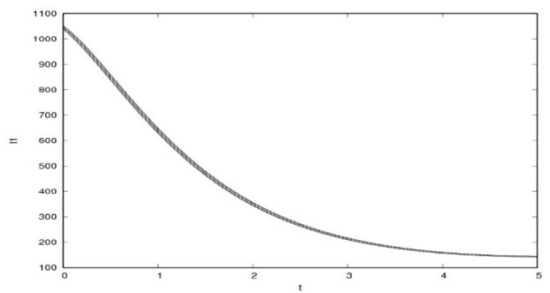

Fig. 13. The result of $I_{1}$ plot in the third scenario with a time of five years.

to 0 . The image shows the number does not reach twice the initial amount, therefore the system is safe. The result of $E_{2}$ plot in the third scenario can be seen in Fig. 12.

The result of the image can $E_{2}$ from start to finish down. The number $E_{2}$ is down from the initial number of 120-160 to close to the value of 0 . The image shows the number does not reach twice the initial amount, therefore the system is safe. The result of $I_{1}$ plot in the third scenario can be seen in Fig. 13.

The result of the image can $I_{1}$ from start to finish down. The $I_{1}$ the number of people down from the beginning was $1140-1150$ to $140-145$. The image shows the number does not reach twice the initial amount, therefore the system is safe. The result $I_{2}$ in third scenario can be seen in Fig. 14 .

The result of the image can be $I_{2}$ the beginning to the end of the drop. The $I_{2}$ is initially 190-200 then dropped until the end of the year to 33-38. The image shows the number does not reach twice the initial amount, therefore the system is safe.

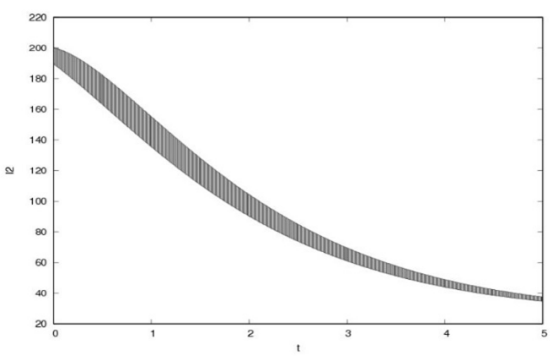

Fig. 14. The result of $I_{2}$ plot in the third scenario with a time of five years.

TABLE I

RUNNING TIME AND VERIFICATION RESULTS OF EACH SCENARIO

\begin{tabular}{c||l||l||l||l}
\hline No & $\begin{array}{l}\text { Initial } \\
\text { condition }\end{array}$ & $\begin{array}{l}\text { Running } \\
\text { time }\end{array}$ & $\begin{array}{l}\text { Verification } \\
\text { time }\end{array}$ & $\begin{array}{l}\text { Verification } \\
\text { results }\end{array}$ \\
\hline \hline 1 & $\begin{array}{l}\text { First } \\
\text { scenario }\end{array}$ & $72 \mathrm{~s}$ & $1.9 \mathrm{~s}$ & safe \\
\hline 2 & $\begin{array}{l}\text { Second } \\
\text { scenario }\end{array}$ & $71 \mathrm{~s}$ & $1.9 \mathrm{~s}$ & safe \\
\hline 3 & $\begin{array}{l}\text { Third sce- } \\
\text { nario }\end{array}$ & $66 \mathrm{~s}$ & $1.9 \mathrm{~s}$ & safe \\
\hline
\end{tabular}

\section{CONCLUSIONS}

The results of safety verification of four subpopulations $E_{1}$, $E_{2}, I_{1}, I_{2}$ are described in Table I. The verification result of these three scenarios is that it is safe or not to be an outbreak of the disease for either the $E_{1}, E_{2}, I_{1}$ sub-population or the $I_{2}$.

\section{REFERENCES}

[1] X. Chen, S. Dutta, and S. Sankaranarayanan, "Formal verification of a multi-basal insulin infusion control model." in ARCH@ CPSWeek, 2017, pp. 75-91.

[2] A. Zuhriah, "Analisa dinamik dari model matematika rekombinasi antara hiv dan virus hepatitis b," Master's thesis, Institut Teknologi Sepuluh Nopember, 2016.

[3] X. Chen, "Reachability analysis of non-linear hybrid systems using taylor models," Ph.D. dissertation, Fachgruppe Informatik, RWTH Aachen University, 2015. 